\title{
Temperature profile and melt depth in laser powder bed fusion of Ti-6Al-4V titanium alloy
}

\author{
Luis E. Criales ${ }^{1} \cdot$ Tuğrul Özel ${ }^{1}$
}

Received: 16 March 2016/ Accepted: 2 August 2017/Published online: 14 August 2017

(c) Springer International Publishing AG 2017

\begin{abstract}
In this paper, the prediction of temperature profile and melt depth for laser powder bed fusion (L-PBF) of Ti-6Al-4V titanium powder material was performed by numerically solving the heat conduction-diffusion equation using a finite difference method. A review of the literature in numerical modeling for laser-based additive metal manufacturing is presented. Initially, the temperature profile along the depth direction into the powder material is calculated for a stationary single pulse laser heat source to understand the transient behavior of the temperature rise during L-PBF. The effect of varying laser pulse energy, average power, and the powder material's density is analyzed. A method to calculate and predict the maximum depth at which localized melting of the powder material occurs is provided.
\end{abstract}

Keywords Lasers · Powder bed fusion - Titanium · Thermal $\cdot$ Modeling

\section{Introduction}

Laser-based additive manufacturing (3-D printing) technology has been rapidly growing and finding applications in various industries including medical implants, automotive and aerospace parts with complex geometries and structures [1]. Specifically, laser powder bed fusion (L-

Tuğrul Özel

ozel@rutgers.edu

1 Manufacturing Automation Research Laboratory, Department of Industrial and Systems Engineering, Rutgers State University of New Jersey, 96 Frelinghuysen Road, Piscataway, NJ 08854, USA
PBF) processes such as direct metal laser sintering $\left(\mathrm{DMLS}^{\mathrm{TM}}\right)$, selective laser melting $\left(\mathrm{SLM}^{\mathrm{TM}}\right)$, LaserCU$\mathrm{SING}^{\mathrm{TM}}$, direct metal production $\left(\mathrm{DMP}^{\mathrm{TM}}\right)$, and laser metal fusion $\left(\mathrm{LMF}^{\mathrm{TM}}\right)$ have been receiving a lot of attention [2]. However, the build part quality and process performance, structural integrity, mechanical properties and related processing times are not at the desired industryready levels. Predictive process modeling and optimization for improved dimensional quality, product reliability, and overall productivity are of great interest to the current, ongoing research efforts [3-7]. This work gives a rapid calculation technique for predicting temperature profile and melt depth of metal powder material during laser melting. It uses pulsed laser heat source to acquire transient temperature behavior and enables studying the influence of process input parameters and powder material properties on the depth of melted material which is very practical for industrial applications.

Selective laser melting is an additive manufacturing process that directly and rapidly fabricates three-dimensional parts by focusing and fully melting metallic powders at selective locations and subsequently allowing for solidification. In laser melting process, the powder material is completely melted and solidified, as opposed to laser sintering processes where metal powder material is sintered, or partially melted $[5,8,11]$. Both groups of processes utilize similar operational set-ups typically requiring a high power fiber laser source, a beam delivery lens system, a scanning mirror, a metal powder supply, a recoater roller or blade, and a build platform, (see Fig. 1). When comparing laser melting to other manufacturing techniques, the L-PBF process has some clear advantages which can be listed as (1) high flexibility in manufacturing complex shapes, (2) quick process set-up avoiding the need for tooling, and (3) high suitability for product customization and use of 


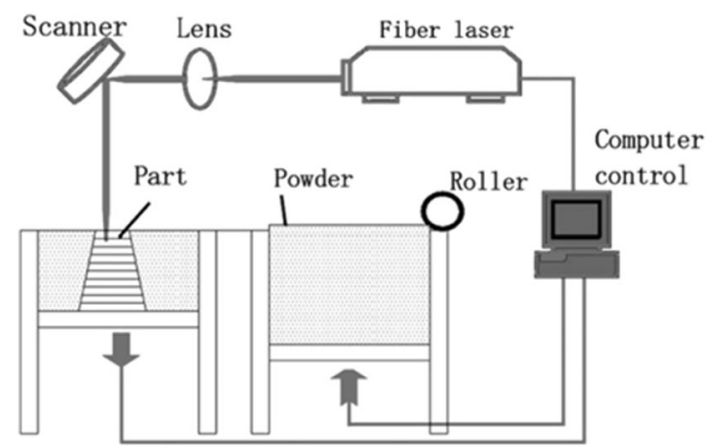

Fig. 1 A common L-PBF process configuration [3]

different powder materials. These advantages allow for quick transition between manufacturing products of different geometries within the same station. The most attractive feature of laser powder bed fusion is the ability to use this process to produce highly complex geometries and structures that would normally not even be feasible using conventional manufacturing processes. However, laser melting has a major disadvantage: the laser heating process is known for its rapid heating times and unstable cooling times, which result in the formation of pores and voids in the microstructure, which often lead to reduced material density and loss of dimensional accuracy and process repeatability.

\section{Literature review}

Temperature distribution possesses a significant factor in the resulting properties of the fabricated components using laser powder bed fusion or laser cladding processes. Therefore, accurately describing the temperature distribution during and after the process is vital to obtaining highquality samples. The temperature distribution for L-PBF can be calculated using either an analytical solution approach or a numerical solution approach. Furthermore, a numerical solution can be obtained two-fold with a finite element analysis (FEA) approach, and by means of applying the finite difference method (FDM). L-PBF and SLS processes use powder material which has different thermal properties when compared to the bulk (fully dense) material. Gusarov et al. [9] established a model to calculate the thermal conductivity of a powder bed based on the molecular structure of the powder. Kolossov et al. [10] formed a thermal model of SLS utilizing finite element method by combining non-linear effects of conductivity and capacity and were able to confirm their results by means of experimental testing on titanium powder material. Patil and Yadava [11] analyzed the effect of laser processing parameters (power, beam size and hatch distance) on the temperature of a single powder layer under SLS and utilized a finite element approach to obtain temperature gradient variations on titanium powder material. Gusarov et al. [12] continued their investigations into SLM by developing a finite element model to analyze the influence of laser scan speed. They concluded that a scan speed range can be found where re-melted lines due to laser scanning are obtained as uniform. Non-uniform lines are undesirable due to their lowered mechanical properties, in what is known as the "balling" effect. Van Elsen et al. [13] provided analytical and computational solutions to the heat equation for moving point and semi-ellipsoidal heat sources. Roberts et al. [14] developed a 3-D finite element solution for computing the transient temperature results for several powder layers. They introduced a unique simulation technique in an attempt to reproduce the complexity created with considering several powder layers. Yin et al. [5] developed a simulation of the temperature distribution for laser micro-sintering (LMS). They analyzed the effect of laser power, beam size, scan speed on the temperature reached and the geometry of the melt pool. Finally, Song et al. [7] developed a FE simulation of the temperature profile to select process parameters in SLM. This group found that the temperature gradient from powder material bed's top surface to the bottom surface is very large. Foroozmehr et al. [17] developed FE simulations considering optical penetration depth of laser in powder bed. Other researchers proposed thermal management systems for laser melting process [18]. In summary, the vast majority of the previous work in the calculation and analysis of the temperature distribution of laser additive manufacturing processes has centered in the development of finite element simulations. Additionally, most of the research efforts have focused on the effect of laser parameters in the process. Even though there are several different solutions presented in literature to the thermal analysis of laser melting processes [11-14, 16, 19-21] little attention has been given to employing analytical thermal modeling numerical solution using FDM analysis techniques, which are ideal for studying and simulating the effect of material properties, controlling and optimization of process parameters.

\section{Materials and methods}

Ti-6Al-4V is a titanium-based two-phase alloy which is very popular in manufacturing applications due to its corrosion resistance, high specific and mechanical strength, super-plasticity, low-weight, and biocompatibility, among many other properties. Its biocompatibility makes it a prime candidate for medical devices and implant applications and it is also widely used in aeronautical, aerospace and automotive applications for its high temperature 
Table 1 Composition of bulk Ti-6Al-4V material

\begin{tabular}{llllllllll}
\hline Element & $\mathrm{Al}$ & $\mathrm{V}$ & $\mathrm{O}$ & $\mathrm{H}$ & $\mathrm{N}$ & $\mathrm{C}$ & $\mathrm{Fe}$ & $\mathrm{Si}$ & $\mathrm{T}$ \\
\hline $\mathrm{wt} \%$ & 6.0 & 4.0 & 0.15 & 0.02 & 0.04 & 0.04 & 0.025 & 0.02 & Balance \\
\hline
\end{tabular}

strength. The compound composition of this material is given in Table 1 as reported by Sun et al. [4].

As explained previously, the biggest disadvantage of the L-PBF process is the difficulty in obtaining maximum possible density and favorable microstructure because of the fast rising temperature of metal powder and the cooling down effects that follow. In particular, the stability, dimensions, and behavior of the melt pool are the determining factors in the porosity and surface roughness of the resulting part. Furthermore, material's physical properties e.g. heat capacity and thermal conductivity vary during the L-PBF process as the powder bed heats up. The temperature-dependent thermal properties of bulk Ti-6Al-4V are summarized in Table 2, as reported by Song et al. [7]. L-PBF of Ti-6Al-4V is a promising advanced manufacturing process because it has the potential to produce samples that satisfy the design requirements for medical device applications, such as complex internal channel geometries and dimensions, the creation of space gradual changing surfaces, and ultrathin walls as well as being suitable for orthodontic and orthopedic customization $[3,6]$. Conventional manufacturing processes are not able to meet these requirements. Most of the existing studies on additive manufacturing of titanium alloys have focused on investment casting beginning with a 3-D printed master pattern, powder deposition, direct sintering or melting using lasers [8]. Additionally, many researchers have opted for the modeling route, attempting to model the L-PBF process and determine temperature profiles and distributions by solving heat transfer equations either analytically and/or numerically (with finite difference method and finite element method analysis).

Powder density is a key material property for proper temperature analysis of laser powder bed fusion or laser cladding processes. Powders consist of a large number of

Table 2 Thermal properties of bulk Ti-6Al-4V [7]

\begin{tabular}{lll}
\hline Temperature $(\mathrm{K})$ & Capacity $(\mathrm{J} / \mathrm{g} \mathrm{K})$ & Conductivity $(\mathrm{W} / \mathrm{cm} \mathrm{K})$ \\
\hline 293 & 0.611 & 0.068 \\
373 & 0.624 & 0.074 \\
473 & 0.653 & 0.087 \\
573 & 0.674 & 0.098 \\
673 & 0.691 & 0.103 \\
773 & 0.703 & 0.118 \\
873 & 0.710 & 0.128 \\
973 & 0.712 & 0.135 \\
\hline
\end{tabular}

very fine particles, whose small size allows them to be compacted or loosened into a wide array of densities. One way to model powder density is by considering it as a fraction of the bulk density $[9,14,16]$ :

$\rho_{\text {powder }}=(1-\tau) \rho_{\text {bulk }}$,

where $\tau$ is the porosity of the powder material, indicating that at $\tau=0$ the powder is fully dense. The bulk density of solid Ti-6Al- $4 \mathrm{~V}$ is $\rho_{\text {bulk }}=4.45 \mathrm{~g} / \mathrm{cm}^{3}$. Therefore, by considering values of $\tau$ between 0 and 1, the effective density of Ti-6Al$4 \mathrm{~V}$ powder can be expressed. As a result of this approach, powder material porosity becomes a process variable. Additionally, thermal properties of the powder material can be obtained from the bulk properties of the material by using the porosity of the powder where $\gamma$ is a model coefficient specific to Ti-6Al-4V as given in $[9,14,16]$.

$k_{\text {powder }}=k_{\text {bulk }}(1-\tau)^{\gamma}$.

One important aspect to note is that most researchers use different laser equipment and a different laser scanning strategy, depending on the resources available. Laser parameters, such as maximum power, wavelength, beam intensity distribution, and spot diameter have a direct influence in the L-PBF process. Additionally, the manner in which the process is conducted, namely the process parameters, will also affect the manufactured product. Important processing parameters include scanning velocity, hatching space, and scanning strategy (also referred to as scanning path) $[4,7]$.

In this paper, the effect of laser parameters in the temperature profile into the depth of powder material versus time is investigated. A temperature profile that shows the depth at which the powder will melt and later solidify with a single pulse can be obtained. By superimposing pulses as the laser heat source moves in the scanning direction, a complete profile of the temperature distribution can be obtained. Additionally, the temperature versus time is determined for a specific location in the powder bed. This will provide a better idea of the behavior of the melted powder pool as a function of time. The decision variables in this case will be the model inputs. These inputs can be both the laser parameters: laser energy, pulse duration, spot size diameter, and scanning speed, and the material properties such as the porosity, or density, of the powder. Once the temperature profile is obtained, the depth of melted material can be predicted, and compared against the desired level. By later applying process optimization techniques, it is possible to determine the variables which 
most affect the temperature profile, and therefore, the maximum depth of melted material. The model to obtain the temperature profile by solving the heat equation is based on finite difference method (FDM), which is presented next.

First, it was assumed that $x$ represents the direction of the moving laser heat source, $y$ is the direction orthogonal to $x$ on the powder bed surface, and $z$ is the direction into the powder material. Also, $t$ will always refer to time unless otherwise noted. The general convection-diffusion equation is given by:

$\frac{\partial \rho u}{\partial t}+\frac{\partial \rho h V}{\partial x}=k\left(\frac{\partial^{2} T}{\partial x^{2}}+\frac{\partial^{2} T}{\partial y^{2}}+\frac{\partial^{2} T}{\partial z^{2}}\right)+\dot{q}$,

where $u$ is the internal energy $(\mathrm{J}), h$ is the enthalpy $(\mathrm{J}), \rho$ is the density $\left(\mathrm{g} / \mathrm{cm}^{3}\right), k$ is the thermal conductivity (W/ $\mathrm{cm} \mathrm{K}), \dot{q}$ is the heat source $\left(\mathrm{W} / \mathrm{cm}^{3}\right), T$ is the material temperature $(\mathrm{K})$, and $V$ is the velocity of the material in fluid phase $(\mathrm{cm} / \mathrm{s})$. In Eq. (3), the change in internal energy as a function of time and the convection are balanced with the heat conduction and the heat source. Given that $\mathrm{d} u=\mathrm{d} h=C \mathrm{~d} T$, we can further simplify this expression by introducing the heat capacity, $C(\mathrm{~J} / \mathrm{g} \mathrm{K})$ :

$\frac{\partial \rho C T}{\partial t}+\frac{\partial \rho C V T}{\partial x}=k\left(\frac{\partial^{2} T}{\partial x^{2}}+\frac{\partial^{2} T}{\partial y^{2}}+\frac{\partial^{2} T}{\partial z^{2}}\right)+\dot{q}$.

Due to the rapid re-solidification nature characteristic of L-PBF, the velocity of the material in fluid phase is very small so it is assumed to be negligible $(V=0)$, so that the convection term drops out resulting in the well-known heat conduction equation. As given in Table 2, the material properties are a function of temperature, which yields:

$\rho(T) C(T) \frac{\partial T}{\partial t}=k(T)\left(\frac{\partial^{2} T}{\partial x^{2}}+\frac{\partial^{2} T}{\partial y^{2}}+\frac{\partial^{2} T}{\partial z^{2}}\right)+\dot{q}$.

An accurate analysis can be obtained by accounting for the change in material properties due to the heating of the powder. Since the objective is to determine the depth at which the powder melts, only the temperature profile in the $z$ direction (depth) is considered. Heat conduction in the $x$-and $y$-directions is not considered due to the limitations that arise from attempting to solve 2D and 3D equations numerically $[12,13,19,21]$. We solved the problem of heat conduction into the powder bed depth direction ( $z$ direction) and neglected heat conduction into the powder bed lateral and longitudinal directions ( $x$ and $y$ directions). Therefore, it is assumed that conduction occurs principally in the $z$ direction:

$\rho(T) C(T) \frac{\partial T}{\partial t}=k(T) \frac{\partial^{2} T}{\partial z^{2}}+\dot{q}$.

To solve this equation numerically, we introduce the Crank-Nicolson scheme of the finite difference method.
The heat equation is a member of the so-called parabolic partial differential equations. This type of equations can be solved using a Crank-Nicolson method which is based on central difference in space and the trapezoidal rule in time providing second-order convergence in time. When considering this 1-D case, the Crank-Nicolson scheme becomes a combination of the forward Euler method at time $t=n$ and the backward Euler method at time $t=n+1$. One key aspect of employing this approach is that for this particular type of differential equation, the Crank-Nicolson method is unconditionally stable. Therefore, very small time and space steps can be considered. For time-dependent derivatives, we utilize the forward derivative, so that the derivate of temperature at depth $(z)$ with respect to time, at $t=i$, is given by:

$\frac{\partial T_{z}^{i}}{\partial t} \approx \frac{T_{z}^{i+1}-T_{z}^{i}}{\Delta t}$

For the second derivative of temperature with respect to the spatial dimension, $z$, the Crank-Nicolson discretization is given by:

$\frac{\partial^{2} T_{z}^{i}}{\partial z^{2}} \approx \frac{T_{z-1}^{i}-2 T_{z}^{i}+T_{z+1}^{i}}{(\Delta z)^{2}}+\frac{T_{z-1}^{i+1}-2 T_{z}^{i+1}+T_{z+1}^{i+1}}{(\Delta z)^{2}}$.

Replacing Eqs. (7) and (8) in the simplified heat equation, Eq. (6), results in a finite difference formulation of the temperature profile:

$$
\begin{aligned}
& \rho(T) C(T)\left(\frac{T_{z}^{i+1}-T_{z}^{i}}{\Delta t}\right) \\
& =k(T)\left(\frac{T_{z-1}^{i}-2 T_{z}^{i}+T_{z+1}^{i}+T_{z-1}^{i+1}-2 T_{z}^{i+1}+T_{z+1}^{i+1}}{(\Delta z)^{2}}\right)+\dot{q} .
\end{aligned}
$$

Solving for $T(z)$ at time $i+1$ gives the implicit equation:

$$
\begin{aligned}
T_{z}^{i+1}= & T_{z}^{i} \\
& +\frac{k \Delta t}{\rho C}\left(\frac{T_{z-1}^{i}-2 T_{z}^{i}+T_{z+1}^{i}+T_{z-1}^{i+1}-2 T_{z}^{i+1}+T_{z+1}^{i+1}}{(\Delta z)^{2}}\right) \\
& +\frac{\dot{q} \Delta t}{\rho C} .
\end{aligned}
$$

The laser heat source is based off the Nd:YAG pulsed laser system [15] with a Gaussian-like laser beam that has a fundamental wavelength of $\lambda=1064 \mathrm{~nm}$, a maximum pulse energy of $E=392 \mathrm{~mJ}$, pulse duration of $t_{\mathrm{p}}=5 \mathrm{~ns}$, pulse frequency $f=10 \mathrm{~Hz}$ and $A=0.25 \mathrm{~cm}^{2}$ spot area. For this simulation, a $5 \times$ focused beam was considered, resulting in a spot size diameter of $d=1.90 \times 10^{-2} \mathrm{~cm}$. By varying the drive voltage, it is possible to modify and control the pulse energy. The laser intensity decays as it 
penetrates the material. This phenomenon can be modeled according to Beer-Lambert's law as follows:

$I(z, t)=I(0, t) e^{-\alpha z}$,

where $I(0, t)$ is the intensity at the surface $\left(\mathrm{W} / \mathrm{cm}^{2}\right)$ and $\alpha$ is the absorption coefficient $(1 / \mathrm{cm})$. The heat source $S(z, t)$ is calculated by taking the negative of the derivative of the intensity with respect to the $z$ direction while considering the effect of the material's reflectivity to laser irradiation. It is assumed that a percentage of the laser energy, given by $R$, is reflected by the material. $R$ is an optical property of the material and will change according to the laser wavelength. For Ti-6Al-4V at near-IR wavelengths, $R=0.75$ $[7,16]$.

$\dot{q}=S(z, t)=\left\{\begin{array}{ll}(1-R) I(0, t) \alpha \mathrm{e}^{-\alpha z}, & t \leq t_{\mathrm{p}} \\ 0, & \text { o.w }\end{array}\right.$.

Two approaches to determine laser intensity are considered. In the first approach we consider the ultra-short transient behavior of temperature rise. In this case, the laser intensity is calculated from the peak power of each pulse, given by:

$P_{\mathrm{p}}=E / t_{\mathrm{p}}$.

The peak intensity can be modeled using the Gaussian function for the corresponding Gaussian beam:

$I(r)=\frac{4 P_{\mathrm{p}}}{\pi d^{2}} \exp \left(-2 \frac{r^{2}}{(d / 2)^{2}}\right)$,

where $r$ is the length from the any given point $(x, y)$ to the laser beam centerline. To determine the intensity at the surface, $I(0, t)$, in Eq. $(11)$, we let $I(0, t)=I(r)$. Then, the heat equation can be solved from the time when the pulse is fired $(t=0)$ to the moment right before the next pulse is fired. The next iteration of the problem is solved by recalculating $I(0, t)$ based on the new distance $r$, using the previous temperature profile as the initial condition, then solving for the temperature distribution again. The process can be repeated until the laser has moved sufficiently away from the point of interest that $I(r)$ is close to 0 . It is clear that the total heat provided by the laser is then a function of the scanning speed. In this particular case, we will focus on the effect of a single pulse irradiation, assuming that $r=0$. Figure 2 shows how peak intensity behaves as the pulse energy is modified.

Furthermore, the intensity profile can be obtained as a function of the depth by plotting Eq. (12) as given in Fig. 3. As expected, the powder material surface receives the laser heat greatly, with little to no heat from the heat source reaching to the bottom plate in the powder bed.

The second approach is to analyze the short transient behavior of temperature rise by considering the average

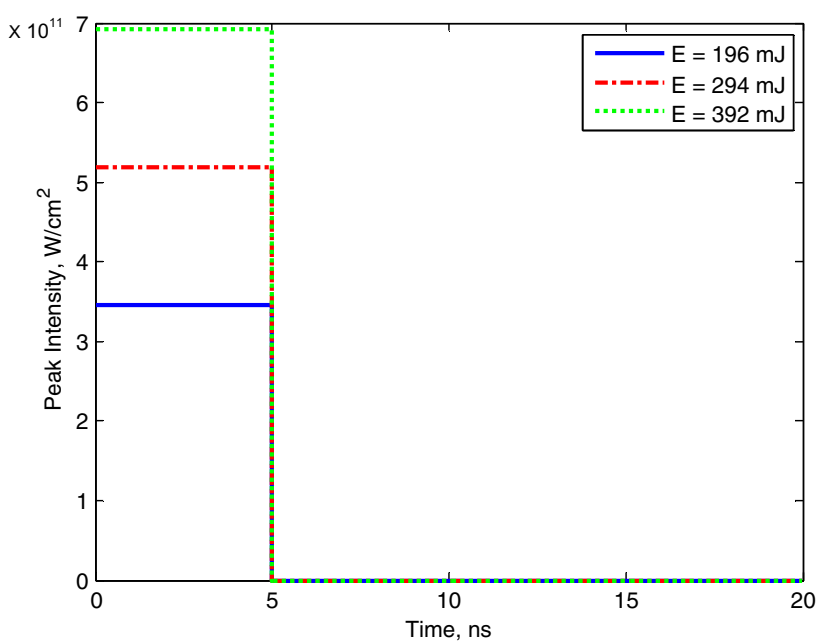

Fig. 2 Peak intensity during a single pulse width at the surface of the powder bed for three energy settings

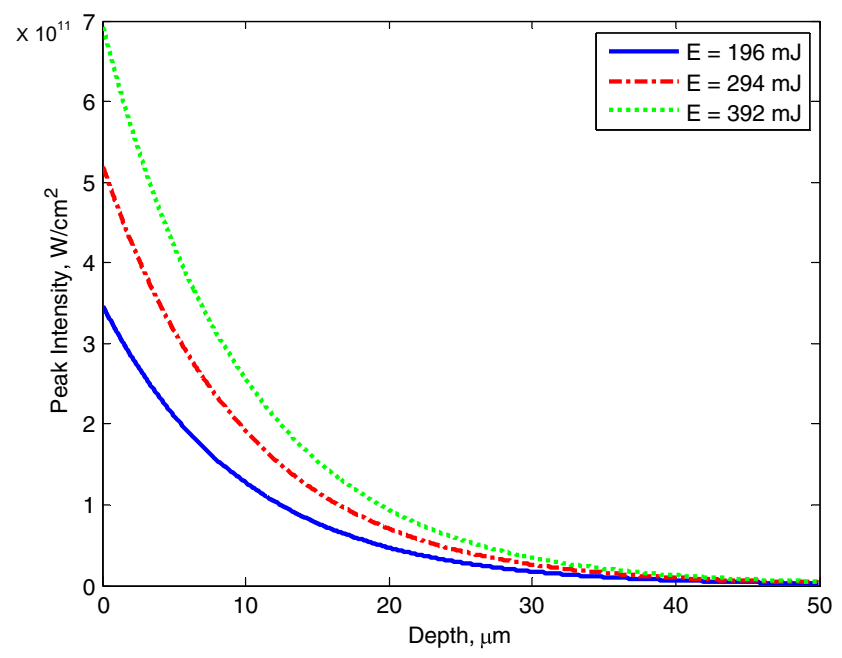

Fig. 3 Peak intensity profile throughout the powder bed at the end of the pulse $(t=5 \mathrm{~ns})$

energy delivered by laser. In this case, the average power is obtained from the laser energy by considering the pulse frequency of the laser, $f$, and can be given as:

$P_{\text {ave }}=E \cdot f$.

The total time that a point of interest is subjected to this average power is determined from the scanning speed and the spot size diameter. Table 3 summarizes the relationship between scanning velocity, $v_{\mathrm{s}}$, and total time the point of

Table 3 Irradiation time for varying scanning velocities

\begin{tabular}{ll}
\hline Scanning velocity, $v_{\mathrm{s}}(\mathrm{cm} / \mathrm{s})$ & Irradiation time, $t_{\mathrm{i}}(\mathrm{ms})$ \\
\hline 10 & 1.90 \\
20 & 0.95 \\
50 & 0.38 \\
\hline
\end{tabular}


interest is subjected to irradiation, $t_{i}$, for $d=1.90 \times 10^{-2}$ $\mathrm{cm}$. It is clearly seen that a faster scanning speed corresponds to a shorter equivalent irradiation time. The latent heat of fusion in laser melting can be defined as specific energy per unit mass required for phase change from solid to liquid. This transformation occurs without a change in temperature, and occurs at the powder material's melting point. For Ti-6Al-4V powder material, this latent heat is $L_{\mathrm{f}}=292 \mathrm{~J} / \mathrm{g}$ [7] and the melting temperature is $T_{\mathrm{m}}=1933 \mathrm{~K}$. There are several ways to incorporate the effect of the latent heat of fusion into the temperature model, either by using a temperature-dependent heat capacity term, or by adding an extra heat sink. The most common method is the apparent heat capacity method, in which a new heat capacity $C_{\text {app }}=C+C_{\mathrm{Lf}}$ is calculated. The heat capacity associated with the latent heat of fusion is given by:

$C_{\mathrm{Lf}}=\frac{L_{\mathrm{f}}}{T_{1}-T_{\mathrm{s}}}$,

where $T_{1}(\mathrm{~K})$ and $T_{\mathrm{s}}(\mathrm{K})$ are the liquidus and solidus temperature of the material, respectively.

\section{Results and discussion}

Numerical analyses have been conducted in order to simulate the temperature profile into the depth of the powder material as well as transient of the temperature rise during pulse width. The 1-D heat conduction equation given by Eq. (4) was solved with Matlab ${ }^{\circledR}$, using the Crank-Nicolson scheme presented in Eq. (8). The simulation code developed takes necessary inputs; physical, thermal, and optical properties of powder material as well as laser pulse energy, power intensity, and pulse width. Table 4 summarizes the thermal and optical material parameters used in this simulation. These parameter values are often used in modeling of Ti-6Al-4V powder material where average particle is considered about $25 \mu \mathrm{m}$ in size. With respect to the numerical analysis of laser-powder material interaction using finite difference method implementation, the parameters given in Table 5 were utilized in the numerical analysis. The choice of simulation parameters, namely the time and space steps, was made based on the achieving

Table 4 Physical, thermal, and optical material parameters

\begin{tabular}{ll}
\hline Parameter & Value \\
\hline Melting temperature, $T_{\mathrm{m}}(\mathrm{K})$ & 1933 \\
Room temperature, $T_{0}(\mathrm{~K})$ & 300 \\
Bulk density, $\rho\left(\mathrm{g} / \mathrm{cm}^{3}\right)$ & 4.45 \\
Reflectivity, $R$ & 0.75 \\
Absorption coefficient, $\alpha(1 / \mathrm{cm})$ & 500,000 \\
\hline
\end{tabular}

Table 5 Finite difference method parameters

\begin{tabular}{ll}
\hline Parameter & Value \\
\hline Total duration, $t_{\max }(\mathrm{s})$ & $20 \times 10^{-9}$ \\
Powder bed thickness, $D(\mathrm{~cm})$ & $100 \times 10^{-4}$ \\
Time step, $\Delta t(\mathrm{~s})$ & $5 \times 10^{-12}$ \\
Space step, $\Delta z(\mathrm{~cm})$ & $0.05 \times 10^{-4}$ \\
Number of time steps & 4000 \\
Number of space steps & 2000 \\
\hline
\end{tabular}

Table 6 Simulation decision variables and resulting maximum melting depth of the powder

\begin{tabular}{llll}
\hline Run & Pulse energy $(\mathrm{mJ})$ & Powder density $\left(\mathrm{g} / \mathrm{cm}^{3}\right)$ & $\begin{array}{l}\text { Max melt } \\
\text { depth }(\mu \mathrm{m})\end{array}$ \\
\hline 1 & 196 & $2.225(50 \%$ dense $)$ & 0 \\
2 & 294 & 2.225 & 0.30 \\
3 & 392 & 2.225 & 3.25 \\
4 & 196 & $1.780(40 \%$ dense $)$ & 0 \\
5 & 294 & 1.780 & 2.60 \\
6 & 392 & 1.780 & 5.45 \\
7 & 196 & $1.112(25 \%$ dense $)$ & 3.25 \\
8 & 294 & 1.112 & 7.30 \\
9 & 392 & 1.112 & 10.20 \\
\hline
\end{tabular}

proper convergence. The underlying assumptions of the model are (1) no heat loss during laser processing, (2) beam intensity follows a Gaussian profile, (3) powder material absorption is not depending on temperature. The initial and boundary conditions applied to solve the heat equation are $T(t=0, z)=300 \mathrm{~K}$ (initial temperature is room temperature), and $T(t, z=D)=300 \mathrm{~K}$ (fixed temperature at the bottom of the sample).

The effect of laser energy and material density on the ultra-short transient temperature profile was analyzed. Table 6 shows how several combinations of pulse energy and powder density affect the maximum depth at which melting of the powder occurs. Figure 4 shows the temperature profile versus time at the powder material surface which has a certain percentage of the bulk density of solid Ti-6Al-4V. It can be seen that the temperature rises consistently from $t=0$ to $5 \mathrm{~ns}$, as the laser pulse acts on the powder bed. After the end of the pulse $(t=5 \mathrm{~ns})$, no heat source acts on the powder bed. The melted material cools as the heat is conducted through the powder bed (Fig. 5).

It should be noted that there is a direct correlation between the maximum depth at which melting of the powder occurs and the maximum temperature, which very close to the surface. If the laser energy is increased indiscriminately, direct thermal ablation of the powder material occurs. Therefore, excessive temperature rise and 

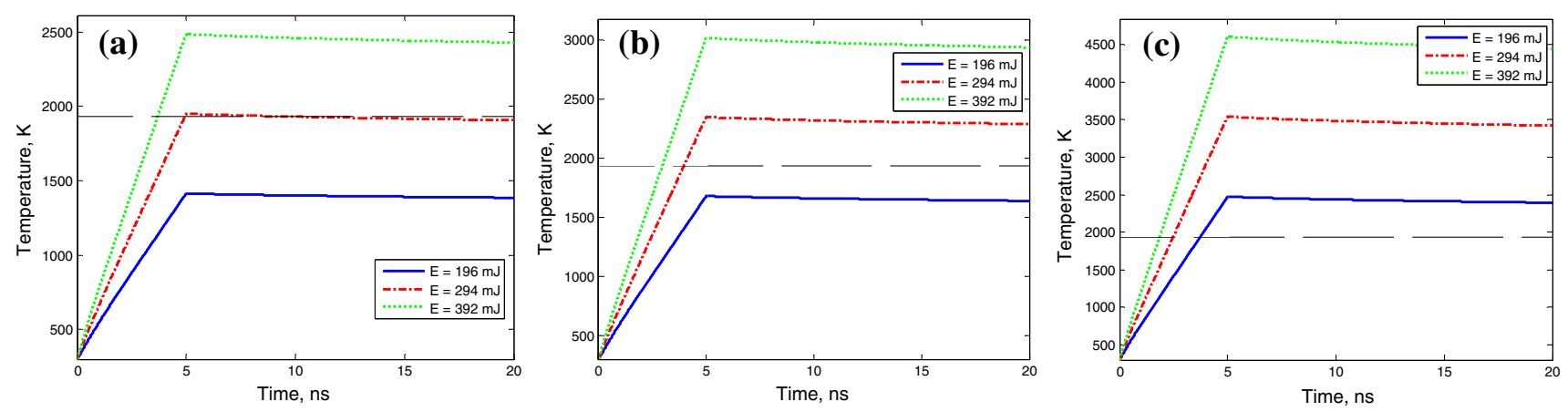

Fig. 4 Temperature vs. time for a 50\% dense, b $40 \%$ dense, c 25\% dense Ti-6Al-4V at three different levels of laser pulse energy by using peak intensity as heat source
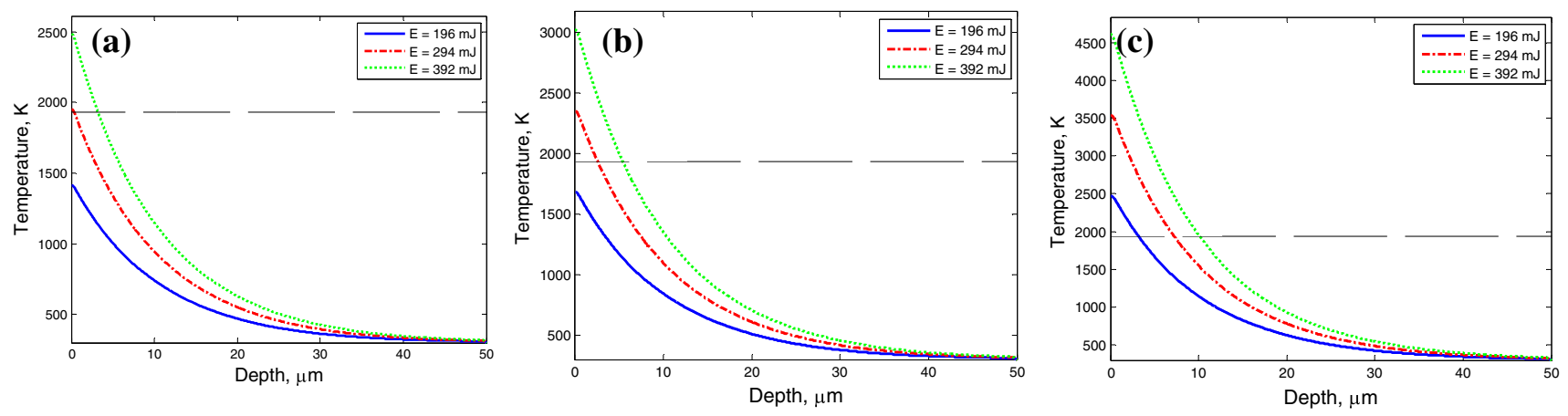

Fig. 5 Temperature vs. depth for a 50\% dense, b $40 \%$ dense, c $25 \%$ dense Ti-6Al-4V at three different levels of laser pulse energy by using peak intensity as heat source

subsequent burning can have adverse effects on the material. Utilizing a high-powered laser may cause gasification of the metal powder, which has adverse effects on part density. In the other hand, a low-energy setting may fail melt the powder depending upon the packing density as can be seen in Fig. 4a, b. It is possible to optimize the laser energy per powder area and volume so that only sufficient amount of melting is achieved Fig. 6 portraits the temperature profile along the depth of the powder bed at the end of the first laser pulse ( $t=5 \mathrm{~ns}$ ).

Figure 6 portrays ultra-short transient temperature behavior as a function of time at three different depths. In Table 5, the maximum depth at which melting occurs had been identified at $3.25 \mu \mathrm{m}$. Figure 7 shows the sharp dropoff in maximum temperature for values of $z$ larger than the maximum depth of melting.

It is clearly seen that higher temperatures are obtained when the porosity of the powder bed is increased. This is clear from Eq. (10), given that the density appears in the denominator. Reducing the density will result in an increase in the temperature gradient. Additionally, a more porous powder bed has a lower thermal conductivity as given by Eq. (2). Therefore, the powder bed will not cool as quickly when the powder bed density is lowered. The sum of these effects results in the temperature rise observed

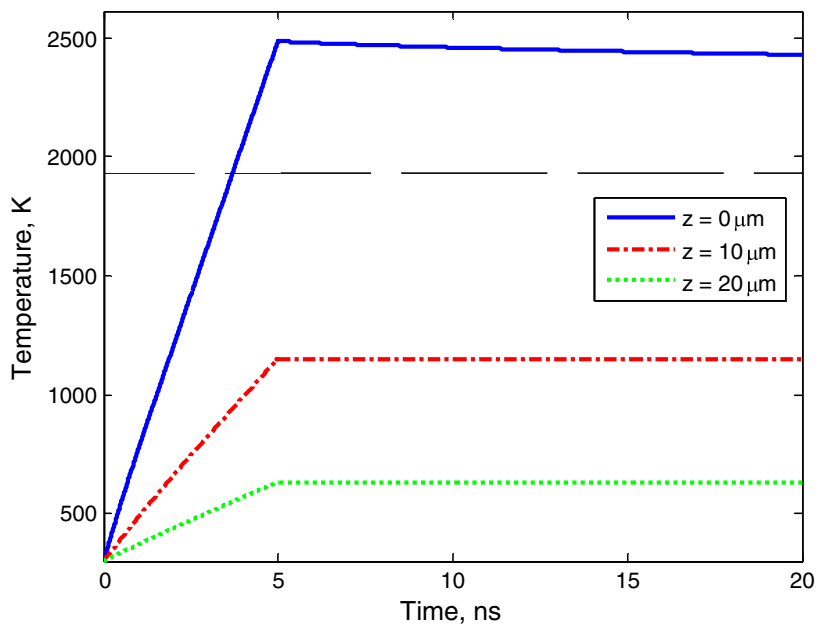

Fig. 6 Ultra-short transient temperature vs. time at several depths for $50 \%$ dense Ti-6Al-4V by using peak intensity as heat source $(E=392 \mathrm{~mJ})$

in Figs. 4 and 5. Additionally, the thermal conductivity of the material increases as the material heats up, accelerating the cooling process. The maximum time considered in the ultra-short transient simulation does not include the entirety of the cooling process, in which the temperature drops below the melting temperature, producing 

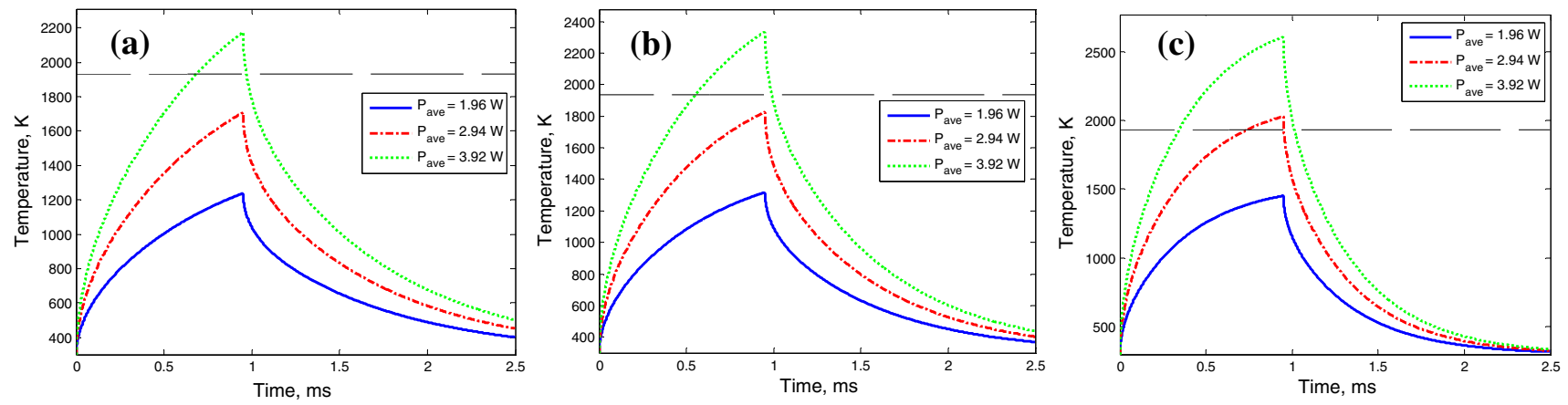

Fig. 7 Temperature vs. time for a 50\% dense, b $40 \%$ dense, c $25 \%$ dense Ti-6Al-4V by using average intensity as heat source

solidification, but rather focuses on the heating process due to the single-pulse heat source. The rapid cooling times result in material porosity and loss of dimensional accuracy, making process repeatability very challenging [16].

The effect of porosity in the short transient temperature behavior was analyzed. For this simulation, the pulse energy was modified to $E=196,294$, and $392 \mathrm{~mJ}$ with a constant frequency $f=10 \mathrm{~Hz}$, resulting in an average power of $P_{\text {ave }}=1.96,2.94$ and $3.92 \mathrm{~W}$, respectively. The scanning speed was fixed at $v_{\mathrm{s}}=20 \mathrm{~cm} / \mathrm{s}$. Figure 7 shows the temperature profile versus time at the powder material surface $(z=0)$ for three different porosity values: 50,45 and $25 \%$ dense powder bed. Finally, the short transient temperature behavior as a function of time at three different depths $(z=0,10$, and $20 \mu \mathrm{m})$ can be seen in Fig. 8 .

\section{Conclusions}

In this paper, a representation of the temperature profile and melt depth in laser fusion of Ti-6Al-4V powder material was obtained. The temperature profile was

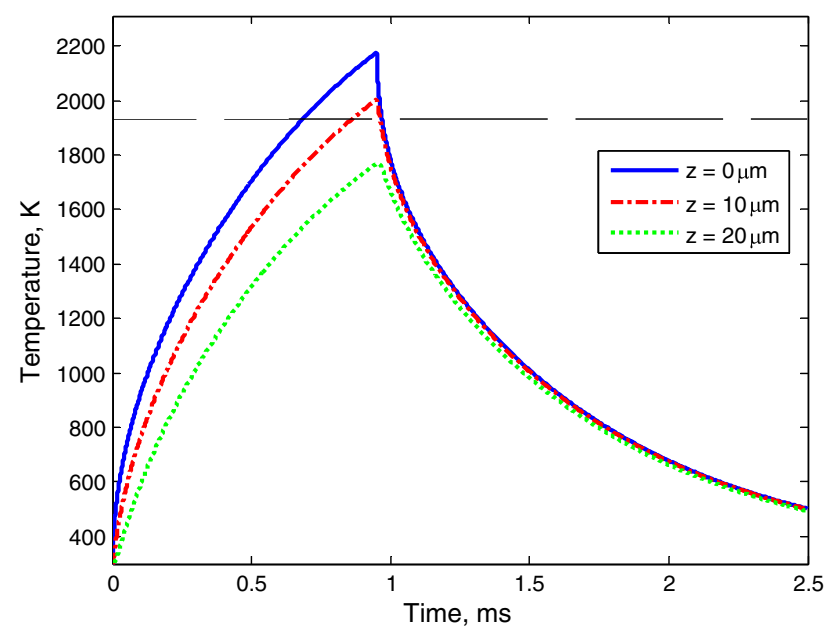

Fig. 8 Ultra-short transient temperature vs. time at several depths for $50 \%$ dense Ti-6Al-4V by using average intensity as heat source numerically solved by using the finite difference method. Additionally, the maximum depth at which melting or cladding of the powder occurs was calculated. Several assumptions to simplify the model were made, such as the use of a single pulse stationary point heat source to obtain the ultra-short transient temperature behavior. With this model, it is possible to understand the influence of process parameters and powder density upon the temperature profile and the depth of melted, sintered or cladding material. In this particular simulation, pulse energy, average power, and powder bed packing density were selected as process decision variables. Through optimization techniques, the best laser and material properties can be determined for optimal laser fusion of metal powders. Material properties can be easily modified to represent other materials of interest besides Ti-6Al-4V.

\section{References}

1. Gibson I, Rosen DW, Stucker B (2009) Additive manufacturing technologies: rapid prototyping to direct digital manufacturing. Springer, US

2. Everton SK, Hirsch M, Stravroulakis P, Leach RK, Clare AT (2016) Review of in situ process monitoring and in situ metrology for metal additive manufacturing. Mater Des 95:431-445

3. Yadroitsev I, Krakhmalev P, Yadroitsava I (2014) Selective laser melting of Ti6Al4V alloy for biomedical applications: temperature monitoring and microstructural evolution. J Alloy Compd 583:404-409

4. Sun J, Yang T, Wang D (2013) Parametric optimization of selective laser melting for forming Ti6Al4V samples by Taguchi method. Opt Laser Technol 49:118-124

5. Yin J, Zhu H, Ke L, Lei W, Dai C, Zuo D (2012) Simulation of temperature distribution in single metallic powder layer for laser micro-sintering. Comput Mater Sci 53:333-339

6. Vandenbroucke B, Kruth J-P (2007) Selective laser melting of biocompatible metals for rapid manufacturing of medical parts. Rapid Prototyp J 13:196-203

7. Song B, Dong S, Liao H (2012) Process parameter selection for selective laser melting of Ti6Al4V based on temperature distribution simulation and experimental sintering. Int $\mathrm{J}$ Adv Manuf Technol 6:967-974 
8. Delgado J, Ciurana J, Rodríguez CA (2012) Influence of process parameters on part quality and mechanical properties for DMLS and SLM with iron-based materials. Int J Adv Manuf Technol 60(5):601-610

9. Gusarov AV, Laoui T, Froyen L, Titov VI (2003) Contact thermal conductivity of a powder bed in selective laser sintering. Int $\mathrm{J}$ Heat Mass Transf 46:1103-1109

10. Kolossov S, Boillat E, Glardon R, Fischer P, Locher M (2004) 3D FE simulation for temperature evolution in selective laser sintering process. Int J Mach Tools Manuf 44:117-123

11. Patil RB, Yadava V (2007) Finite element analysis of temperature distribution in single metallic powder layer during metal laser sintering. Int J Mach Tools Manuf 47:1069-1080

12. Gusarov AV, Yadroitsev I, Bertrand Ph, Smurov I (2007) Heat transfer modelling and stability analysis of selective laser melting. Appl Surf Sci 254:975-979

13. Van Elsen M, Baelmans M, Mercelis P, Kruth J-P (2007) Solutions for modeling moving heat sources in a semi-infinite medium and applications to laser material processing. Int $\mathbf{J}$ Heat Mass Transf 50:4872-4882

14. Roberts IA, Wang CJ, Esterlein R, Stanford M, Mynors DJ (2009) A three-dimensional finite element analysis of the temperature field during laser melting of metal powders in additive layer manufacturing. Int J Mach Tools Manuf 49:916-923

15. Criales LE, Orozco PF, Medrano A, Rodriguez CR, Özel T (2015) Effect of fluence and pulse overlapping on fabrication of microchannels in PMMA/PDMS via UV laser micromachining: modeling and experimentation. Mater Manuf Processes 30(7):890-901

16. Hussein A, Hao L, Yan C, Everson R (2013) Finite element simulation of the temperature and stress fields in single layers built without-support in selective laser melting. Mater Des 52:638-647

17. Foroozmehr A, Badrossamay M, Foroozmehr E (2016) Finite element simulation of selective laser melting process considering optical penetration depth of laser in powder bed. Mater Des 89:255-263

18. Ilin A, Logvinov R, Kulikov A, Prihodovskya A, Xu H, Ploshikhin V, Günther B, Bechmann F (2014) Computer aided optimisation of the thermal management during laser beam melting process. Phys Procedia 56:390-399

19. Gusarov AV, Smurov I (2009) Two-dimensional numerical modelling of radiation transfer in powder beds at selective laser melting. Appl Surf Sci 255:5595-5599

20. Han L, Liou FW, Musti S (2005) Thermal behavior and geometry model of melt pool in laser material process. Trans ASME J Heat Transf 127(9):1005-1014

21. Verhaeghe F, Craeghs T, Heulens J, Pandalaers L (2009) A pragmatic model for selective laser melting with evaporation. Acta Mater 57:6006-6012 